\title{
Superconductors with broken time-reversal symmetry: Spontaneous magnetization and quantum Hall effects
}

\author{
Baruch Horovitz ${ }^{1,2}$ and Anatoly Golub ${ }^{1}$ \\ ${ }^{1}$ Department of Physics and ${ }^{2}$ Ilze Katz center for nanotechnology, \\ Ben-Gurion University of the Negev, Beer-Sheva 84105, Israel
}

\begin{abstract}
Broken time reversal symmetry (BTRS) in $d+i d^{\prime}$ as well as in $d+i s$ superconductors is studied and is shown to yield current carrying surface states. We evaluate the temperature and thickness dependence of the resulting spontaneous magnetization and show a marked difference between weak and strong BTRS. We also derive the Hall conductance which vanishes at zero wavevector $q$ and finite frequency $\omega$, however at finite $q, \omega$ it has an unusual structure. The chirality of the surface states leads to quantum Hall effects for spin and heat transport in $d+i d^{\prime}$ superconductors.
\end{abstract}

PACS numbers: 74.20Rp, 74.25.Ha, 74.25.Fy

\section{INTRODUCTION}

Recent data on the high $T_{c}$ superconductor $\mathrm{YBa}_{2} \mathrm{Cu}_{3} \mathrm{O}_{x}$ (YBCO) has supported the presence of broken time reversal symmetry (BTRS) ${ }^{1.2 .3}$. A sensitive probe of BTRS are Andreev surface states. For a d wave with time reversal symmetry bound states at zero energy are expected for a surface parallel to the nodes (i.e. a (110) surface in YBCO). When BTRS is present, by either a complex order parameter or by an external magnetic field, the bound states shift to a finite energy. Indeed tunnelling data usually shows a zero bias peak which splits in an applied field; the splitting is nonlinear in the magnetic field, indicating a proximity to a BTRS state 2.4 . In fact, in some samples tunnelling data shows a splitting even without an external field ${ }^{1.2}$, consistent with BTRS; the splitting increases with increasing overdoping 2.5 ,

Further support for a spontaneous BTRS state are spontaneous magnetization data as observed in $\mathrm{YBCO}^{3}$, setting in abruptly at $T_{c}$ and being almost temperature $(T)$ independent below $T_{c}$. The phenomenon has been attributed to either a $d_{x^{2}-y^{2}}+i d_{x y}$ state $\left(d+i d^{\prime}\right)$ or to formation of $\pi$ junctions. No microscopic reason was given, however, for the spontaneous magnetization being independent of both $T$ and of film thickness ${ }^{3}$.

It has been shown theoretically that BTRS can occur locally in a $d_{x^{2}-y^{2}}$ superconductor near certain surfaces 6.7 .8 .9 leading to either $d+i d^{\prime}$ or $d+i s$ states with surface currents. The onset of such BTRS is expected to be below $T_{c}$ and therefore does not correspond to the spontaneous magnetization data ${ }^{3}$. We note that in response to an external magnetic field the surface states are paramagnetic and compete with Meissner currents. This effect has been proposed to account for a minimum in the magnetic penetration length ${ }^{10}$. In fact, it was proposed that this paramagnetic effect leads to spontaneous currents and BTRS in a pure $d_{x^{2}-y^{2}}$ state 11.12 . The onset of this BTRS is much below $T_{c} \frac{12}{2}$ and therefore does not correspond to the data ${ }^{3}$.

Of further theoretical interest is the relation of the BTRS state to quantum Hall systems with a variety of Hall effects13.14.15.16.17. In particular a finite charge hall conductance has been suggested ${ }^{13}$, though this has been questioned ${ }^{16}$.

In the present work we expand our earlier work ${ }^{18}$ and study variety of phenomena related to surface currents. In section II we show that bulk $d+i d^{\prime}$ state has surface states with finite surface currents; a similar situation was found for the bulk p wave state $\frac{19}{}$. We also consider a $d+i$ s state which has surface currents only on the (110) surface. In section III we evaluate the spontaneous magnetization and show that for $d+i d^{\prime}$ it is dominated by (100) surfaces; for thin films it increases with the ratio $\Delta^{\prime} / \Delta\left(\Delta\right.$ and $\Delta^{\prime}$ are the amplitudes of $d_{x^{2}-y^{2}}$ and $d_{x y}$, respectively) while for thick films it has a maximum at $\lambda / \xi^{\prime} \approx 1$ where $\xi^{\prime}=v_{F} / \Delta^{\prime}$ with $v_{F}$ the Fermi velocity and $\lambda$ is the penetration length, i.e. at $\Delta^{\prime} / \Delta \approx 0.01$ for YBCO. We show that for weak BTRS, $\lambda / \xi^{\prime}<1$, the spontaneous magnetization is $T$ and thickness independent, while for strong BTRS thickness and $T$ dependence may occur. For the sample of Ref 3 we estimate $\Delta^{\prime} / \Delta \approx 10^{-4}$, i.e. weak BTRS. In section IV we consider a surface approach for the quantum Hall effect, showing quantization for spin and thermal Hall conductances for the $d+i d^{\prime}$ state. We also derive in section $\mathrm{V}$ the effective action in the bulk and identify the Hall coefficient which has an unusual wavevector and frequency dependence.

\section{SURFACE STATES}

We present here the Bogoliubov de-Gennes (BdG) equations for quasiparticles in a bulk $d+i d^{\prime}$ or $d+i s$ states in presence of a boundary and study the resulting surface states. We consider first a $d+i d^{\prime}$ state where the order 
parameter is

$$
\Delta\left(\hat{p}_{x}, \hat{p}_{y}\right)=\Delta\left(\hat{p}_{x}^{2}-\hat{p}_{y}^{2}\right) / k_{F}^{2}+i \Delta^{\prime} \hat{p}_{x} \hat{p}_{y} / k_{F}^{2}
$$

where $\hat{\mathbf{p}}=-i \hbar \nabla$ is the momentum operator and $k_{F}$ is the Fermi momentum. The quasiparticles are represented by an electron-hole Nambu spinor

$$
\Psi(\mathbf{r})=\left(\begin{array}{c}
\Psi_{\uparrow}(\mathbf{r}) \\
\Psi_{\downarrow}^{\dagger}(\mathbf{r})
\end{array}\right)
$$

and are described by the following mean field Hamiltonian (see appendix A)

$$
\hat{\mathcal{H}}=\frac{1}{2 m}\left[\left(i \boldsymbol{\nabla}+\frac{e}{c} \tau_{3} \mathbf{A}(\mathbf{r})\right)^{2}-k_{F}^{2}\right] \tau_{3}+\left(\begin{array}{cc}
0 & \Delta\left(\hat{p}_{x}, \hat{p}_{y}\right) \\
\Delta^{*}\left(\hat{p}_{x}, \hat{p}_{y}\right) & 0
\end{array}\right) e^{-i \theta(\mathbf{r}) \tau_{3}}
$$

where $m$ is the electron mass and $\tau_{i}$ are the Pauli matrices. We assume here that $|\nabla \theta| \ll k_{F}$ so that the issue of gauge invariance in the interaction term can be avoided (appendix A). Rotating by the unitary transformation $\Psi(\mathbf{r}) \rightarrow \exp \left[i \tau_{3} \theta(\mathbf{r}) / 2\right] \Psi(\mathbf{r})$ yields

$$
\hat{\mathcal{H}}=\frac{1}{2 m}\left(-\nabla^{2}-k_{F}^{2}\right) \tau_{3}+\frac{1}{2 m} \mathbf{p} \cdot\left(\boldsymbol{\nabla} \theta-\frac{2 e}{c} \mathbf{A}(\mathbf{r})\right)+\left(\begin{array}{cc}
0 & \Delta\left(\hat{p}_{x}, \hat{p}_{y}\right) \\
\Delta^{*}\left(\hat{p}_{x}, \hat{p}_{y}\right) & 0
\end{array}\right)
$$

where $\mathbf{A}$ is kept to first order.

We consider a vacuum-superconductor boundary at $\mathrm{x}=0$, and assume for now that $\Delta, \Delta^{\prime}$ are constants at $x>0$ and vanish at $x<0$. For $\Delta>\Delta^{\prime}$ this corresponds to a (100) surface; to describe a (110) surface $\Delta$ and $\Delta^{\prime}$ need to be interchanged. The spinor wavefunctions for the up and down component of Eq. (2), respectively, $u(\mathbf{r})=$ $u \exp \left[i f x+i k_{y} y\right]$ and $v(\mathbf{r})=v \exp \left[i f x+i k_{y} y\right]$ with eigenvalues $\epsilon$ satisfy the BdG equations

$$
\begin{aligned}
\left(f^{2}-k_{F}^{2}+k_{y}^{2}-2 m \tilde{\epsilon}\right) u+2 m \Delta\left(f, k_{y}\right) v & =0 \\
\left(-f^{2}+k_{F}^{2}-k_{y}^{2}-2 m \tilde{\epsilon}\right) v+2 m \Delta^{*}\left(f, k_{y}\right) u & =0
\end{aligned}
$$

where $\tilde{\epsilon}=\epsilon+(e / m c) k_{y} A_{y}(x), \mathbf{A}$ has only an $A_{y}$ component consistent with a current in the $y$ direction and $\nabla \theta=0$. This Doppler shift assumes that $A_{y}(x)$ is slowly varying on the scale $k_{F}^{-1}$ so that a local eigenevalue $\tilde{\epsilon}$ can be defined. Define $k=+\sqrt{k_{F}^{2}-k_{y}^{2}}$, then $f$ has two surface solutions with $\Im f>0$

$$
\begin{aligned}
& f_{1}=k+i \frac{m}{k} \sqrt{\left|\Delta\left(k, k_{y}\right)\right|^{2}-\tilde{\epsilon}^{2}} \\
& f_{2}=-k+i \frac{m}{k} \sqrt{\left|\Delta\left(-k, k_{y}\right)\right|^{2}-\tilde{\epsilon}^{2}}
\end{aligned}
$$

where the replacement $\Delta\left(f, k_{y}\right) \rightarrow \Delta\left( \pm k, k_{y}\right)$ is valid for $|\Delta|, \tilde{\epsilon} \ll k_{F}^{2} / 2 m$. The eigenvectors are

$$
\begin{gathered}
v_{1}=-i \frac{\sqrt{\left|\Delta\left(k, k_{y}\right)\right|^{2}-\tilde{\epsilon}^{2}}+i \tilde{\epsilon}}{\Delta\left(k, k_{y}\right)} u_{1} \\
v_{2}=\quad i \frac{\sqrt{\left|\Delta\left(-k, k_{y}\right)\right|^{2}-\tilde{\epsilon}^{2}}-i \tilde{\epsilon}}{\Delta\left(-k, k_{y}\right)} u_{2}
\end{gathered}
$$

We assume specular reflection which preserves $k_{y}$ but mixes these two solutions so that at $x=0$ the wavefunctions vanish. A linear combination for which both spinor components vanish at $x=0$, i.e. $\alpha u_{1}+\beta u_{2}=\alpha v_{1}+\beta v_{2}=0$ yields $v_{1} / u_{1}=v_{2} / u_{2}$, hence an equation for the eigenvalues

$$
\frac{i \tilde{\epsilon}+\sqrt{\left|\Delta\left(+k, k_{y}\right)\right|^{2}-\tilde{\epsilon}^{2}}}{-i \tilde{\epsilon}+\sqrt{\left|\Delta\left(-k, k_{y}\right)\right|^{2}-\tilde{\epsilon}^{2}}}=-\frac{\Delta\left(+k, k_{y}\right)}{\Delta\left(-k, k_{y}\right)}
$$

Its solutions are readily seen to be $\tilde{\epsilon}=-\operatorname{sign}\left(k_{y}\right) \Delta\left(k^{2}-k_{y}^{2}\right) / k_{F}^{2}$. In terms of the incidence angle $\zeta, k_{y}=k_{F} \sin \zeta$, $k=k_{F} \cos \zeta$, the eigenvalues are

$$
\epsilon_{\zeta}=-\operatorname{sign}(\zeta) \Delta \cos (2 \zeta)-\frac{e}{c} v_{F} A_{y} \sin \zeta
$$




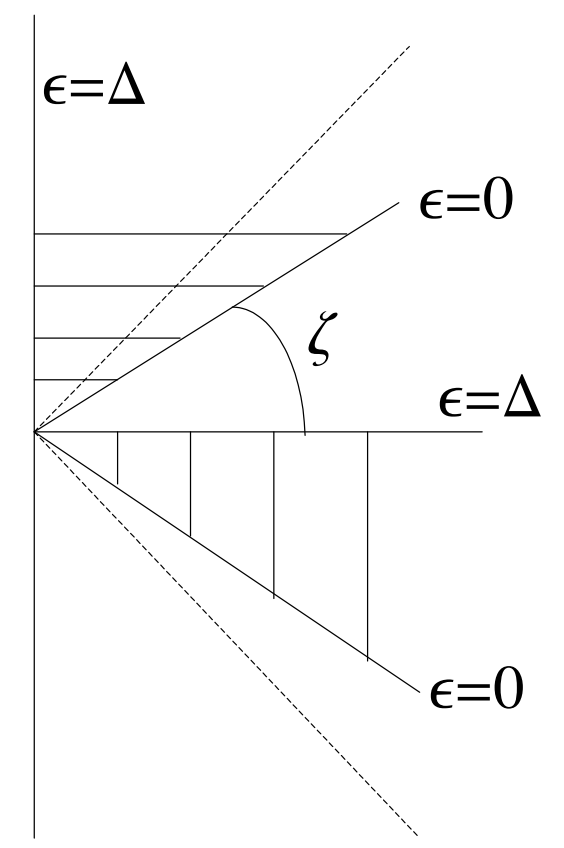

FIG. 1: Surface (vertical line) and angle $\zeta$ where the spectrum is $\epsilon=0$ (full lines); in the absence of $A_{y}$ these lines would be at $\zeta= \pm \pi / 4$ (dashed lines). The range for which $\epsilon>0$ is shown as the hatched area; the spectra spans the range $\epsilon=0$ up to $\epsilon=\Delta$ as shown

Note that the spectrum is not symmetric in $k_{y}$ or in $\zeta$ (it is in fact antisymmetric) resulting in a finite surface current. Fig. 1 shows the angle $\zeta$ where $\epsilon_{\zeta}=0$ (full lines) and the range for which $\epsilon_{\zeta}>0$. The velocities $\partial \epsilon_{\zeta} / \partial k_{y}$ are positive for both $\pm k_{y}$ branches, i.e. the surface states are chiral. This property leads to quantization of Hall effects, as discussed in section IV.

We note that self consistency would imply that $\Delta^{\prime}=0$ at $x=0$; the eigenfunctions would then be $\sim \exp \left[-\int_{0}^{x} \Delta^{\prime}\left(x^{\prime}\right) d x^{\prime}|\sin \zeta| / v_{F}\right]$, resulting in a very similar dependence on $\xi^{\prime}$. Note also that quasiparticles in the bulk have a spectral gap $\left|\Delta\left(k, k_{y}\right)\right|$ which for any given $\zeta$ is higher than the surface states $\epsilon_{\zeta}$ (neglecting the Doppler shift). Impurities, however, may destroy $k_{y}$ conservation and scatter high energy $\left(>\Delta^{\prime}\right)$ surface states into degenerate bulk states. When impurity scattering is essential (e.g. section IV) our results apply only when these excitations can be neglected, e.g. at $T<\Delta^{\prime}$. This restriction is not needed at the (110) surface where the whole surface spectrum is $<\Delta^{\prime}$, i.e. below the lowest bulk state.

The decay length of the surface states becomes, using Eqs. (6] 9), $(\Im f)^{-1}=\left[(m / k) \sqrt{|\Delta|^{2}-\tilde{\epsilon}^{2}}\right]^{-1}=\xi^{\prime} /|\sin \zeta|$ with $\xi^{\prime}=v_{F} / \Delta^{\prime}$. Since $\left|u_{i}\right|=\left|v_{i}\right|, i=1,2$ (Eq. 17) the normalized eigenfunctions are

$$
\begin{aligned}
& u_{\zeta}(\mathbf{r})=\sqrt{\frac{2|\sin \zeta|}{\xi^{\prime} L_{y}}} \sin k x e^{i k_{y} y-x|\sin \zeta| / \xi^{\prime}} \\
& v_{\zeta}(\mathbf{r})=-\operatorname{sign}\left(k_{y}\right) u(\mathbf{r})
\end{aligned}
$$

where $L_{y}$ is the length of the surface. It is remarkable that $\left|u_{\zeta}(\mathbf{r})\right|=\left|v_{\zeta}(\mathbf{r})\right|$ for all $\zeta$, i.e. for all energies of the surface states, implying maximal electron-hole mixing. As noted above, a (110) surface has the same solution (10) with $\xi^{\prime}$ replaced by $\xi=v_{F} / \Delta$.

We note that in general the spinor Eq. (2) can be decomposed in terms of eigenoperators $\eta_{\zeta \uparrow}, \eta_{\zeta \downarrow}$ where

$$
\left(\begin{array}{c}
\Psi_{\uparrow}(\mathbf{r}) \\
\Psi_{\downarrow}^{\dagger}(\mathbf{r})
\end{array}\right)=\sum_{\zeta}\left(\begin{array}{cc}
u_{\zeta}(\mathbf{r}) & -v_{\zeta}^{*}(\mathbf{r}) \\
v_{\zeta}(\mathbf{r}) & u_{\zeta}^{*}(\mathbf{r})
\end{array}\right)\left(\begin{array}{c}
\eta_{\zeta \uparrow} \\
\eta_{\zeta \downarrow}^{\dagger}
\end{array}\right)
$$


leading to the diagonal Hamiltonian

$$
\hat{\mathcal{H}}=\sum_{\zeta} \int d x \epsilon_{\zeta}\left[\eta_{\zeta \uparrow}^{\dagger} \eta_{\zeta \uparrow}+\eta_{\zeta \downarrow}^{\dagger} \eta_{\zeta \downarrow}-1\right]\left[|u(x)|^{2}+|v(x)|^{2}\right]
$$

with $\epsilon_{\zeta}$ being $x$ dependent via the Doppler shift. The spectrum has exact particle-hole symmetry, i.e. for each eigenvector $u, v$ with eigenvalue $\epsilon$ there is an eigenvector $-v *, u *$ with eigenvector $-\epsilon$. The form Eq. (12) incorporates, however, both $\pm \epsilon$ states and its sum is therefore restricted to $\epsilon_{\zeta} \geq 0$.

We consider next a $d+i s$ state at a (110) surface with an order parameter

$$
\Delta\left(\hat{p}_{x}, \hat{p}_{y}\right)=\Delta \hat{p}_{x} \hat{p}_{y} / k_{F}^{2}+i \Delta_{s} .
$$

Eq. (8) has then the solution $\tilde{\epsilon}=\operatorname{sign}\left(k_{y}\right) \Delta_{s}$, i.e.

$$
\epsilon=\operatorname{sign} \zeta \Delta_{s}-\frac{e}{c} v_{F} A_{y} \sin \zeta
$$

Positive eigenvalues are now at $k_{y} \geq 0$ (for weak Doppler effect $\frac{e}{c} v_{F}\left|A_{y}\right|<\Delta_{s}$ ) with a weak dispersion due to the Doppler term. Note in particular that the spectrum has a gap, i.e. no $\epsilon=0$ states; hence to probe these states one needs either high voltage or high temperature $T>\Delta_{s}$. This $d+i$ s state corresponds to a (110) surface at which it breaks both parity and time reversal. At a (100) surface the state $d+i s$ state is symmetric under reflection and in fact has no surface bound states. Hence tunnelling data at the (100) may distinguish between $d+i d^{\prime}$ and $d+i s$ states, i.e. the $d+i d^{\prime}$ state shows a weak structure at a bias $\approx \Delta$ while a $d+i s$ state has no effect at all. The magnetization data ${ }^{3}$ shows an effect for both (110) and (100) surfaces, supporting a $d+i d^{\prime}$ state for YBCO.

\section{SPONTANEOUS MAGNETIZATION}

The $d+i d^{\prime}$ or $d+i s$ order parameters break both time reversal invariance and reflection along the surface, hence they allows surface currents $(d+i s$ refers to (110) only). The current density parallel to a surface (the $y$ direction) and the charge density are,

$$
\begin{aligned}
j_{\text {edge }}(x) & =\frac{-i \hbar e}{2 m d} \sum_{s}\left[\left\langle\Psi_{s}^{\dagger}(\mathbf{r}) \frac{\partial}{\partial y} \Psi_{s}(\mathbf{r})\right\rangle-h . c .\right]=\frac{-2 \hbar e}{m d} \sum_{\zeta} k_{y}|u(\mathbf{r})|^{2} \tanh \left(\frac{\epsilon_{\zeta}}{2 T}\right) \\
n_{\text {edge }} & =\frac{e}{d} \sum_{s}\left\langle\Psi_{s}^{\dagger}(\mathbf{r}) \Psi_{s}(\mathbf{r})\right\rangle=\frac{2 e}{d} \sum_{\zeta}\left|u_{\zeta}(\mathbf{r})\right|^{2}
\end{aligned}
$$

where $d$ is the interlayer spacing, $\left\langle\gamma_{\zeta, s}^{\dagger} \gamma_{\zeta, s}\right\rangle=\left[1+\exp \left(\epsilon_{\zeta} / T\right)\right]^{-1}$ and $\left|u_{\zeta}(\mathbf{r})\right|=\left|v_{\zeta}(\mathbf{r})\right|$ were used. The expression for $j_{\text {edge }}$ can also be obtained from Eq. (12) by $j_{\text {edge }}=c \delta H / \delta A_{y}(x)$. In addition to the explicit $T$ dependence in (15) the order parameters are $T$ dependent as $\Delta \approx \Delta_{0} \sqrt{\tau}, \Delta^{\prime} \approx \Delta_{0}^{\prime} \sqrt{\tau}$ where $\tau=\left(T_{c}-T\right) / T_{c}$; hence $\xi \approx \xi_{0} / \sqrt{\tau}, \xi^{\prime} \approx \xi_{0}^{\prime} / \sqrt{\tau}$.

In principle the current has also a diamagnetic term $(e / c) n_{\text {edge }}(x) A_{y}(x)$; the ratio of this term to the London term $\left(c / 4 \pi \lambda^{2}\right) A_{y}(x)$ is $1 /\left[\left(k_{F} \xi_{0}^{\prime}\right)^{2} \tau\right]$ where $\tau=\left(T_{c}-T\right) / T_{c}$. Hence the effect of this diamagnetic current is small except very near $T_{c}$, i.e. for $\tau>\left(k_{F} \xi_{0}^{\prime}\right)^{-2}$ (or $\tau>\left(k_{F} \xi_{0}\right)^{-2}$ for the (110) surface). In the range where the order parameter fluctuations exceed its mean value mean field breaks down; this range, which is between $\tau<1 / k_{F} \xi_{0}$ in 2-dimensions and $\tau<\left(1 / k_{F} \xi_{0}\right)^{4}$ in 3 -dimensions is excluded in our analysis.

We consider first $d+i d^{\prime}$; the factor $k_{y} \tanh \left(\epsilon_{\zeta} / 2 T\right)$ is symmetric in $k_{y}$, therefore within the integration in Eq. (15) on the $\epsilon_{\zeta} \geq 0$ range (Fig. 1) the $\zeta<0$ segment can be shifted into a $\zeta>0$ one so that a complete $(0, \pi / 2)$ range results. In terms of the density $n=k_{F}^{2} / 2 \pi d$ and the $T=0$ penetration length $\lambda_{0}\left(\lambda \approx \lambda_{0} / \sqrt{\tau}\right)$ where $\lambda_{0}^{-2}=4 \pi n e^{2} / m c^{2}=2 k_{F}^{2} e^{2} / m c^{2} d$ we obtain

$$
\begin{aligned}
\frac{4 \pi}{c} j_{\text {edge }}(x) & =\frac{2 \phi_{0}}{\pi \xi^{\prime} \lambda_{0}^{2}} \int_{0}^{\pi / 2} d \zeta \cos \zeta \sin ^{2} \zeta e^{-2 x \sin \zeta / \xi^{\prime}} \tanh \left[\frac{\Delta \cos 2 \zeta+(e / c) v_{F} \sin \zeta A_{y}(x)}{2 T}\right] \\
n_{\text {edge }}(x) & =\frac{e k_{F}}{\pi d \xi^{\prime}} \int_{0}^{\pi / 2} d \zeta \cos \zeta \sin \zeta e^{-2 x \sin \zeta / \xi^{\prime}}
\end{aligned}
$$

where the rapid oscillatory $\sin ^{2} k x$ is replaced by its average $\frac{1}{2}$. Note that for $\Delta=0$ or $\Delta^{\prime}=0$ all angles $\zeta$ are allowed in the solution of Eq. (8) and then the current vanishes. This demonstrates that BTRS leads to current carrying 
surface states. We note also that if the Doppler shift $\sim A_{y}(x)$ is ignored the integrated current $j_{\text {edge }}(x)$ vanishes, unlike the p wave case ${ }^{19}$.

The response of the condensate to $j_{\text {edge }}$ involves the London terms as well as coupling to the scalar potential at the surface; the latter terms are small as $1 / k_{F} \xi_{0}$ at low $T$ or vanish at $T \rightarrow T_{c}$ (see Appendix C). London's equation with $j_{\text {edge }}(x)$ as a source term is then,

$$
-\nabla^{2} A_{y}(x)=\left[-\left(1 / \lambda^{2}\right) A_{y}(x)+(4 \pi / c) j_{\text {edge }}(x)\right] \theta(x)
$$

where $\theta(x)$ is a step function. This assumes a thick film, i.e. no dependence on the $\mathrm{z}$ direction; the thin film limit is considered below. For a thick film the condition of no external field at $x \rightarrow-\infty$ implies $H_{y}(0)=0$. Eq. (17) is then solved by the Greens' function

$$
G\left(x, x^{\prime}\right)=-(\lambda / 2)\left[\exp \left(-\left|x-x^{\prime}\right| / \lambda\right)+\exp \left(-\left|x+x^{\prime}\right| / \lambda\right)\right]
$$

which satisfies the boundary condition $\left.\partial_{x} G\left(x, x^{\prime}\right)\right|_{x=0}=0$ equivalent to $H_{y}(x=0)=\left.\partial_{x} A_{y}\right|_{x=0}=0$. $A_{y}(x)$ then satisfies an integral equation

$$
\begin{gathered}
A_{y}(x)=\frac{\phi_{0} \lambda}{\pi \xi^{\prime} \lambda_{0}^{2}} \int_{0}^{\pi / 2} d \zeta \cos \zeta \sin ^{2} \zeta \int_{0}^{\infty} \quad d x^{\prime} \tanh \left[\frac{\Delta \cos 2 \zeta+(e / c) v_{F} \sin \zeta A_{y}\left(x^{\prime}\right)}{2 T}\right]\left[e^{-\left|x-x^{\prime}\right| / \lambda}+\right. \\
\left.e^{-\left|x+x^{\prime}\right| / \lambda}\right] e^{-2 x^{\prime} \sin \zeta / \xi^{\prime}}
\end{gathered}
$$

The doppler shift, as shown below, is significant only very near $T_{c}$ or at very low temperatures. Neglecting first the Doppler shift and at $T \rightarrow T_{c}$ Eq. (19) becomes

$$
A_{y}(0)=\left(2 \phi_{0} \lambda \Delta / \pi \lambda_{0}^{2} T_{c}\right) \int_{0}^{\pi / 2} d \zeta \cos \zeta \sin ^{2} \zeta \cos 2 \zeta\left(2 \sin \zeta+\xi^{\prime} / \lambda\right)^{-1}
$$

The total spontaneous flux is $\Phi=A_{y}(0) L_{y}$ where $L_{y}$ is the length of the boundary. We consider $2 \phi_{0} L_{y} \lambda \Delta / \pi \lambda_{0}^{2} T_{c}$ as a flux unit, e.g. for $L_{y}=2 \mathrm{~cm}^{3}$ and typical YBCO parameters it is $\approx 10^{5} \phi_{0}$. This flux unit is weakly temperature dependent since $\lambda \Delta \approx \lambda_{0} \Delta_{0}$ is finite at $T \rightarrow T_{c}$. The ratio $\tilde{\Phi}=-\Phi /\left(2 \phi_{0} L_{y} \lambda \Delta / \pi \lambda_{0}^{2} T_{c}\right)$ is plotted in Fig. 2 ; it varies between $\lambda / 12 \xi^{\prime}$ at $\lambda \ll \xi^{\prime}$ (weak BTRS) to $\xi^{\prime} / 12 \lambda$ at $\lambda \gg \xi^{\prime}$ (strong BTRS) with a maximum of 0.014 at $\lambda \approx \xi^{\prime}$. For a (110) surface replacing $\xi^{\prime}$ by $\xi$ (considering only $\xi \ll \lambda$ ) we obtain $\tilde{\Phi}=\xi \Delta^{\prime} / 12 \lambda \Delta$, much smaller than for a (100) surface. The reason for the dominance of the (100) surface is the steeper spectra $\epsilon \sim \Delta$ for this case. The result that $\Phi$ is weakly temperature dependent at $T \rightarrow T_{c}$ is consistent with the spontaneous magnetization data ${ }^{3}$; more details on the data follow in section VI.

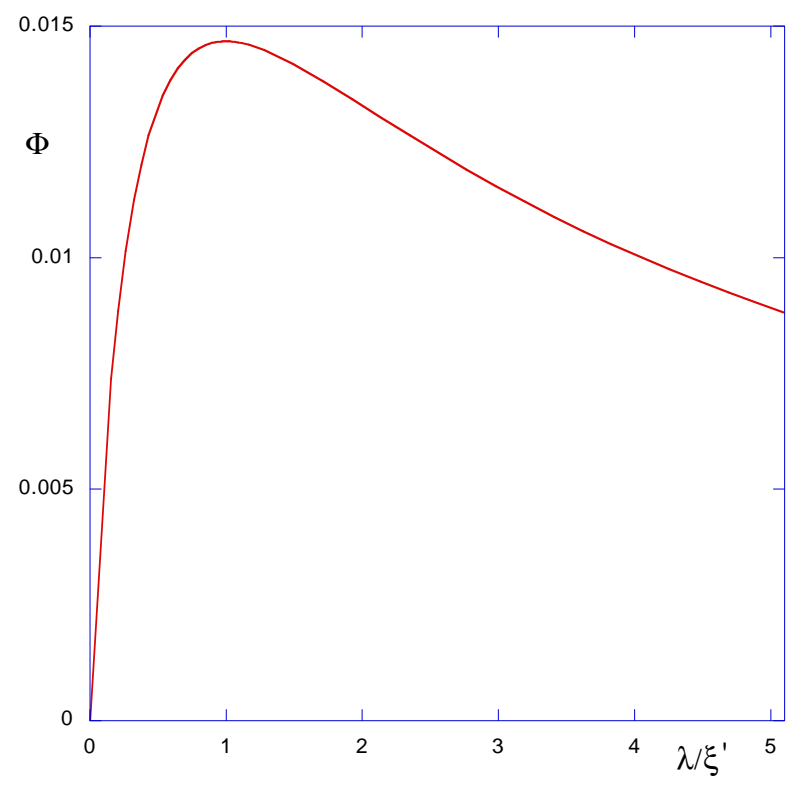

FIG. 2: Spontaneous flux for a (100) boundary in thick films $\left(\xi^{\prime}<\bar{d}\right)$ in units of $2 \phi_{0} L_{y} \lambda \Delta / \pi \lambda_{0}^{2} T_{c}$. 
TABLE I: Spontaneous flux $\tilde{\Phi}$ [flux in units of $-\phi_{0} L_{y} \lambda \Delta /\left(\pi \lambda_{0}^{2} T_{c}\right)$ ] for various surfaces, weak $\left(\xi^{\prime} \gg \lambda\right)$ or strong BTRS $\left(\xi^{\prime} \ll \lambda\right)$ and various temperature limits. Comments: (i) If thin film is not specified the entry corresponds to thick films with thickness $\bar{d} \gg \lambda, \xi^{\prime}\left[(100)\right.$ surface] or $\bar{d} \gg \lambda, \xi\left[(110)\right.$ surface]. (ii) All entries correspond to $d+i d^{\prime}$ except the $d+i s$ one which refers only to (110) surface; for thin films it is the same as (110) $d+i d^{\prime}$ (except a factor -5 in the $T \rightarrow T_{c}$ line). (iii) $T \rightarrow T_{c}$ entries for $\tilde{\Phi}$ exclude paramagnetic anomaly regions which are given in the last column. (iv) BTRS which sets in at a temperature $T_{c}^{\prime} \ll T_{c}$ has $\tilde{\Phi}$ values corresponding to modified temperature intervals. The only paramagnetic anomalies in this case are $(100) T<T_{s}$ and (110) thin film. (v) The fluctuation region (e.g. $\tau \lesssim 1 / k_{F} \xi_{0}$ in 2-dimensions) is excluded, hence the region $\tau=\left(T_{c}-T\right) / T_{c}$ in the last column is relevant only if it is a larger one.

\begin{tabular}{|c|c|c|c|c|}
\hline geometry & temperature & case $T_{c}^{\prime} \ll T_{c}$ & $\tilde{\Phi}$ & paramagnetic anomaly \\
\hline$(100) \xi^{\prime} \gg \lambda$ & $T \rightarrow T_{c}$ & & $\frac{\lambda}{15 \xi^{\prime}}$ & $\tau \approx\left(\frac{\Delta^{\prime}}{\Delta}\right)^{2}$ \\
\hline & $T \ll T_{c}$ & $T<T_{c}^{\prime}$ & $(2-\sqrt{2}) \frac{\lambda T_{c}}{6 \xi^{\prime} \Delta_{0}}$ & \\
\hline$(100) \xi^{\prime} \ll \lambda$ & $T \rightarrow T_{c}$ & & $\frac{\xi^{\prime}}{12 \lambda}$ & $\tau \approx\left(\frac{\xi}{\lambda}\right)^{2}$ \\
\hline & $T \ll T_{c}$ & $T<T_{c}^{\prime}$ & $(\sqrt{2}-1) \frac{\xi^{\prime} T_{c}}{2 \lambda \Delta_{0}}$ & \\
\hline$(110)$ & $T \rightarrow T_{c}$ & $T \rightarrow T_{c}^{\prime}$ & $\frac{\xi^{2}}{12 \lambda \xi^{\prime}}$ & $\tau \approx\left(\frac{\xi}{\lambda}\right)^{2}$ \\
\hline & $T_{s}<T \ll T_{c}, \Delta^{\prime}$ & $T_{s}<T \ll T_{c}^{\prime}$ & $(\sqrt{2}-1) \frac{\xi T_{c}}{2 \lambda \Delta_{0}}$ & \\
\hline & $T<T_{s}$ & $T<T_{s}$ & $\frac{T_{c}}{\Delta_{0}}$ & $T_{s} \approx \frac{\xi}{\lambda} T_{c}$ \\
\hline (100) thin film & $T \rightarrow T_{c}$ & & $\frac{\lambda}{15 \xi^{\prime}}$ & $\tau \approx\left(\frac{\Delta^{\prime}}{\Delta}\right)^{2}$ \\
\hline & $T \ll T_{c}$ & $T<T_{c}^{\prime}$ & $(2-\sqrt{2}) \frac{\lambda T_{c}}{\xi^{\prime} \Delta_{0}}$ & \\
\hline (110) thin film & $T \rightarrow T_{c}$ & $T \rightarrow T_{c}^{\prime}$ & $-\frac{2 \lambda_{0} T_{c}}{15 \xi^{\prime} \Delta_{0}}$ & Doppler dominated \\
\hline & $T \ll T_{c}$ & $T \ll T_{c}^{\prime}$ & $\pm \frac{2 \lambda T_{c}}{3 \xi \Delta_{0}}$ & Doppler dominated \\
\hline$d+i s$ & $T \rightarrow T_{c}$ & $T \rightarrow T_{c}^{\prime}$ & $\frac{\xi}{2 \xi_{a}}$ & $\tau \approx\left(\frac{\xi}{\lambda}\right)^{2}$ \\
\hline & $T \ll T_{c}, \Delta_{s}$ & $T \ll T_{c}^{\prime}$ & $\frac{T_{c}}{\Delta_{0}}$ & $T_{s} \approx \frac{\xi}{\lambda} T_{c}$ \\
\hline
\end{tabular}

At low temperatures $T \ll T_{c}$ the result for the (100) surface is of the same order as that near $T_{c}$ while for the (110) $\Phi$ is enhanced upon cooling, becoming at $T \ll T_{c}, \Delta^{\prime}$ of the order of $\tilde{\Phi} \approx \xi / \lambda$. The various limiting forms of $\tilde{\Phi}$ are collected in table I.

We consider next the results with the Doppler shift. For the (100) surface and $\xi^{\prime} \gg \lambda$ the kernel $G\left(x, x^{\prime}\right)$ is localized at $x \approx x^{\prime}$ so that $A_{y}\left(x^{\prime}\right)$ can be replaced by $A_{y}(x)$ in Eq. (19). Near $T_{c}$ we expand the tanh and obtain a term which modifies $1 / \lambda^{2}$, i.e.

$$
\left[\frac{d^{2}}{d x^{2}}-\frac{1}{\lambda^{2}}+\frac{\Delta^{\prime}}{2 T \lambda_{0}^{2}}\right] A_{y}(x)=\frac{4 \pi}{c} j_{y}^{(0)}(x)
$$

where $j_{y}^{(0)}(x)$ is the current in the absence of the Doppler term. Very neat $T_{c}$, the effective London length $\lambda_{e f f}$ where $1 / \lambda_{\text {eff }}^{2}=1 / \lambda^{2}-\Delta^{\prime} / 2 T \lambda_{0}^{2}$ becomes imaginary so that there is no Meissner effect, i.e. a magnetic field can penetrate into the bulk. Hence a sharp sign change of $A_{y}(0)$ from paramagnetic to diamagnetic is expected at $\tau \approx\left(\Delta^{\prime} / \Delta\right)^{2}$. For $T \ll T_{c}$ we obtain from $j_{y}^{(0)}$ that $\frac{e}{c} v_{F} A_{y}(0) \approx \Delta^{\prime} \ll \Delta$, i.e. the Doppler shift is negligible.

For the (100) surface and $\xi^{\prime} \ll \lambda$ the $x^{\prime}$ integration in Eq. (19) is limited to $\xi^{\prime}$, hence we can replace $A_{y}\left(x^{\prime}\right)$ by $A_{y}(0)$ to yield

$$
A_{y}(x)=\frac{\phi_{0} \lambda}{\pi \lambda_{0}^{2}} \int_{0}^{\pi / 2} d \zeta^{\prime} \cos \zeta \sin \zeta \tanh \left[\frac{\Delta \cos 2 \zeta+\frac{e}{c} v_{F} \sin \zeta A_{y}(0)}{2 T}\right] e^{-x / \lambda} .
$$

At $T \rightarrow T_{c}$ this becomes

$$
\left(1-\frac{\hbar v_{F} \lambda}{3 T \lambda_{0}^{2}}\right) A_{y}(0)=A_{y}^{(0)}
$$

hence the response changes sign at $\tau \lesssim(\xi / \lambda)^{2}$. For $T \ll T_{c}$ the Doppler term can be neglected $\frac{e}{c} v_{F} A_{y}(0) \approx$ $\Delta_{0} \xi \xi^{\prime} / \lambda^{2} \ll \Delta_{0}$. For the (110) surface the form (23) applies with $\xi^{\prime} \rightarrow \xi$ (considering always $\xi \ll \lambda$ ) which does not affect the left hand side of Eq. [23); hence a paramagnetic anomaly at $\tau \approx(\xi / \lambda)^{2}$. 


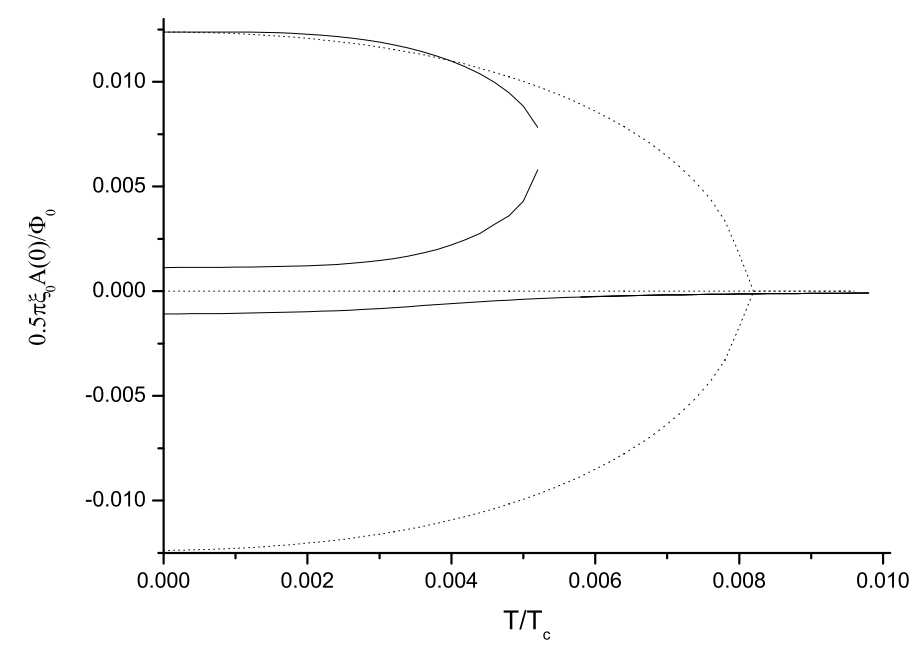

FIG. 3: Spontaneous flux at a (110) surface for $\Delta^{\prime}=0$ (dotted lines) showing a critical temperature $T_{s}$. For $\Delta^{\prime} / \Delta=0.01$ it shows enhancement below $T_{s}$.

A remarkable feature of Eq. (22) is that it allows spontaneous magnetization for the (110) surface even if $\Delta^{\prime}=0$, as studied earlier ${ }^{11,12}$. The critical temperature can be deduced from Eq. (22) (with $\Delta \rightarrow \Delta^{\prime}$ ) by assuming a small probing $\Delta^{\prime}$ and look for the $A_{y}$ response, which from Eq. (23) diverges at $T_{s}=v_{F} / 3 \lambda_{0} \approx(\xi / \lambda) T_{c} \ll T_{c}$. Furthermore, at $T=0$ Eq. (22) yields

$$
A_{y}(0)=\frac{\phi_{0}}{\pi \lambda_{0}} \operatorname{sign}\left[A_{y}(0)\right]
$$

hence a spontaneous magnetization flux of $\pm \phi_{0} L_{y} / \pi \lambda_{0}$.

In Fig. 3 we show the low temperature form of $A_{y}(0)$ for (110). For $\Delta^{\prime}=0$ it shows a spontaneous magnetization (dotted lines) below a critical temperature, while for $\Delta^{\prime} \neq 0$ it shows enhancement near $T_{s}$ where it joins one of the low $T$ branches. In comparison the (100) flux depends weakly on temperature and is much stronger than that of (110) at least at high temperatures.

We consider next the thin film case, for which London's equation is

$$
-\nabla^{2} A(x, z)=\bar{d}\left[-\frac{1}{\lambda^{2}} A_{y}(x)+\frac{4 \pi}{c} j(x)\right] \theta(x) \delta(z)
$$

where $\bar{d}$ is the film thickness. Assuming that one can Fourier transform $A(x, z)$ into $A(q, k)$, integration of the $k$ dependence $\sim\left(q^{2}+k^{2}\right)^{-1}$ yields for $A_{y}(x)=A_{y}(x, z=0)$

$$
A_{y}(x)=\int \frac{d q}{2 \pi|q|} \int d x^{\prime} e^{i q\left(x-x^{\prime}\right)} \bar{d}\left[-\frac{1}{\lambda^{2}} A_{y}(x)+\frac{4 \pi}{c} j(x)\right] \theta(x) .
$$

The $q$ integration then yields

$$
A_{y}(x)-A_{y}(0)=\int_{0}^{\infty} d x^{\prime} \ln \left|\frac{x-x^{\prime}}{x^{\prime}}\right| \bar{d}\left[-\frac{1}{\lambda^{2}} A_{y}(x)+\frac{4 \pi}{c} j(x)\right]
$$

implying a slow decay of $A_{y}(x)$. While a solution for $A_{y}(x)$ appears difficult to obtain, the value of $A_{y}(0)$ is readily noticed from the boundary condition. The absence of external filed requires a finite $H_{y}(x=0)$ for the thin film geometry. Hence to avoid divergence of $d A /\left.d x\right|_{x=0}$ where

$$
\left.\frac{d A(x)}{d x}\right|_{x=0}=\int_{0}^{\infty} \frac{d x^{\prime}}{x^{\prime}} \bar{d}\left[\frac{1}{\lambda^{2}} A_{y}(x)-\frac{4 \pi}{c} j(x)\right]
$$




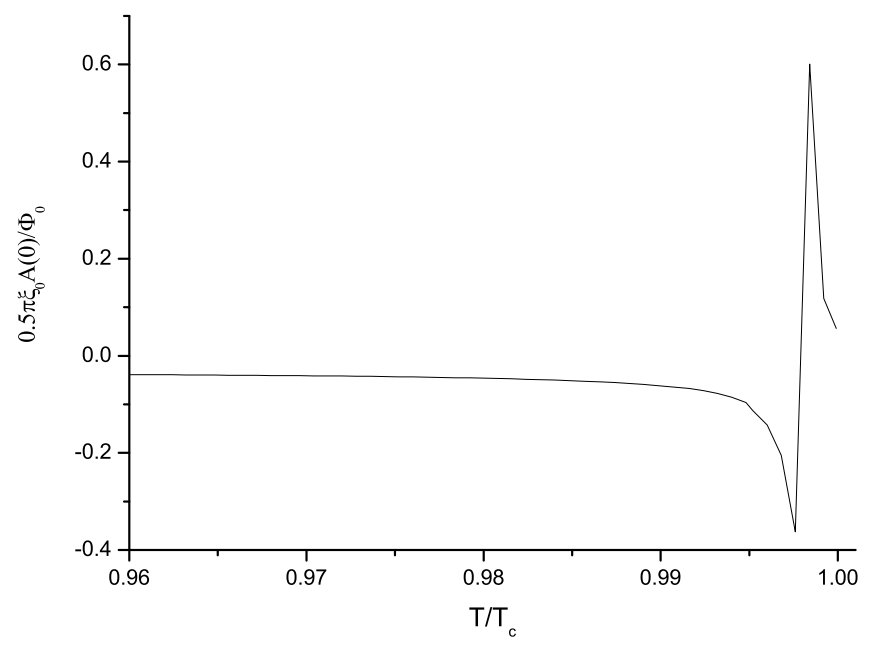

FIG. 4: Doppler induced paramagnetic anomaly near $T_{c}$ for thin films with a (100) surface and $\Delta^{\prime} / \Delta=0.1$

one must have $A_{y}(0)=\lambda^{2} \frac{4 \pi}{c} j(0)$, i.e.

$$
A_{y}(0)=\frac{2 \lambda^{2} \phi_{0}}{\pi \xi^{\prime} \lambda_{0}^{2}} \int_{0}^{\pi / 2} d \zeta^{\prime} \cos \zeta \sin ^{2} \zeta \tanh \left[\frac{\Delta \cos 2 \zeta+\frac{e}{c} v_{F} \sin \zeta A_{y}(0)}{2 T}\right]
$$

which interestingly has the same form as Eq. (22) except that here it is valid for all $\xi^{\prime}$. In particular, when the Doppler shift can be neglected we obtain $\tilde{\Phi}=\lambda / 15 \xi^{\prime}$ which in Fig. 2 is the tangent line to the thick film curve at the origin. Hence we can define two regimes: Weak BTRS with $\lambda / \xi^{\prime}<1$ where the spontaneous flux is $T$ and $\bar{d}$ independent, and strong BTRS with $\lambda / \xi^{\prime}>1$ where film thickness matters, with the thin film showing a stronger effect. For strong BTRS $T$ dependence is induced as $\xi^{\prime}<\bar{d}$ changes to the thin film case $\xi^{\prime}>\bar{d}$ as $T \rightarrow T_{c}$.

Consider now the Doppler shift for thin films; expansion near $T_{c}$ yields $1 / \lambda_{\text {eff }}=1 / \lambda^{2}-\Delta^{\prime} / 2 T \lambda_{0}^{2}$ which as above changes sign at $\tau \approx\left(\xi / \xi^{\prime}\right)^{2}$, i.e. a paramagnetic anomaly. This temperature is the same as for the thick film case except that here it is valid also for $\xi^{\prime}<\lambda$. Hence for $\Delta^{\prime} / \Delta=0.1$ we can have an anomaly at an accessible temperature of $\left(T_{c}-T\right) / T_{c} \approx 10^{-2}$, as shown in Fig. 4 . For $T \ll T_{c}$ the Doppler effect is small.

For a (110) surface the scales of $A_{y}(x)$ are $\lambda, \xi$, hence the the thin film situation applies when $\bar{d} \ll \xi$, which is more difficult to achieve. Near $T_{c}$ we obtain $A_{y}(0)=\frac{2 \phi_{0}}{15 \pi \xi} \Delta^{\prime} \Delta>0$ which is paramagnetic, while at $T \ll T_{c}$ we have $A_{y}(0)= \pm \frac{4 \phi_{0}}{3 \pi \xi_{0}}$. A spontaneous flux even with $\Delta^{\prime}=0$ is possible also here as in the (110) thick film case.

All the various forms for the magnetization and Doppler effects are summarized in table I. The table also considers the possibility that BTRS sets in at a temperature $T_{c}^{\prime} \ll T_{c}$. In this case $\xi^{\prime}$ diverges at $T_{c}^{\prime}$ so that near $T_{c}^{\prime}$ we have $\xi^{\prime} \gg \lambda$ while at $T \ll T_{c}^{\prime}$ also $\xi^{\prime}<\lambda$ is possible, resulting in a temperature dependent $\tilde{\Phi}$ for this strong BTRS case. is

Finally we consider the $d+i s$ case. Here only (110) is relevant and $\xi \ll \lambda$ for high $T_{c}$ materials. The edge current

$$
\frac{4 \pi}{c} j_{e d g e}(x)=\frac{2 \phi_{0}}{\pi \xi \lambda_{0}^{2}} \int_{0}^{\pi / 2} d \zeta \cos \zeta \sin ^{2} \zeta e^{-2 x \sin \zeta / \xi} \tanh \left[\frac{\Delta_{s}+(e / c) v_{F} \sin \zeta A_{y}(x)}{2 T}\right] .
$$

Near $T_{c}$ Eq. (20) applies with $\Delta \cos 2 \zeta$ replaced by $\Delta_{s}$ and $\xi^{\prime} \rightarrow \xi$, hence $\tilde{\Phi}=\Delta_{s} / 2 \Delta=\xi / 2 \xi_{s}$. At low temperatures the tanh is replaced by 1 , leading to $\tilde{\Phi}=T_{c} / \Delta$. The various results are given in table I.

\section{QUANTUM HALL EFFECTS - A SURFACE APPROACH}

In this section we study a surface formulation of quantum Hall effects (QHE). For the usual charge conduction, in the absence of an external field, and given that the surface fields decay in the bulk (Meissner effect as shown in 
section III), Ampére's law implies a zero net current, i.e. a net Hall conductance $\sigma_{x y}=0$. We focus therefore on spin and thermal Hall effects. These were shown to be quantized first by a network model simulations 14 and then by the relation to edge states 15 . The $d+i s$ state has no surface states near $\epsilon=0$, hence no Hall effect within linear response; there may be a response when the voltage exceeds $\approx 2 \Delta_{s} / e$. We consider therefore in this section only the $d+i d^{\prime}$ case.

The main ingredient is the chiral nature of the surface states. These states have two branches whose spectra vanishes at $k_{y}=Q \equiv \pm k_{F} / \sqrt{2}$. Linearizing near this point the spectrum for $k_{y}=q \pm Q$ is

$$
\epsilon=v q
$$

where $v=\sqrt{8} \Delta / k_{F}$ for a (100) surface while $v=\sqrt{8} \Delta^{\prime} / k_{F}$ for the (110) (up to a small Doppler shift; the actual value of $v$ is not important for the eventual result for the Hall conductance). We wish to rewrite the surface modes in terms of two branches $\eta_{1, q}, \eta_{2, q}$ where

$$
\begin{aligned}
\eta_{1, q} & =\eta_{q+Q \uparrow} \\
\eta_{2,-q} & =\eta_{q+Q \downarrow}^{\dagger} \\
\eta_{2, q} & =\eta_{q-Q \uparrow} \\
\eta_{1,-q} & =-\eta_{q-Q \downarrow}^{\dagger}
\end{aligned}
$$

with the \pm signs needed for continuity (see below). Thus, instead of two $q>0$ branches with two $\uparrow, \downarrow$ spins we have now two branches, each of a single degree of freedom, with both $q>0$ and $q<0$. The transformation of Eq. (11) with the eigenfunctions (10) is

$$
\left(\begin{array}{c}
\Psi_{\uparrow}(\mathbf{r}) \\
\Psi_{\downarrow}^{\dagger}(\mathbf{r})
\end{array}\right)=\sum_{q>0}\left\{f_{q}(x)\left(\begin{array}{cc}
1 & 1 \\
-1 & 1
\end{array}\right)\left(\begin{array}{c}
\eta_{1, q} e^{i(Q+q) y} \\
\gamma_{2,-q} e^{-i(Q+q) y}
\end{array}\right)+f_{-q}(x)\left(\begin{array}{cc}
1 & -1 \\
1 & 1
\end{array}\right)\left(\begin{array}{c}
\eta_{2, q} e^{-i(Q-q) y} \\
\gamma_{1,-q} e^{i(Q-q) y}
\end{array}\right)\right\}
$$

where the two terms correspond to the two branches near $\pm Q$ and

$$
f_{q}(x)=\sqrt{\frac{2|Q+q|}{\xi^{\prime} L_{y} k_{F}}} \sin \left(x \sqrt{k_{F}^{2}-(Q+q)^{2}}\right) e^{-x|Q+q| / \xi^{\prime} k_{F}} .
$$

Note e.g. that the $Q \pm q$ Fourier components of $\Psi_{\uparrow}(\mathbf{r})$ are $-\eta_{1, q}$ for both $\pm q$ so that the \pm signs in Eq. (32) are needed for continuity of the Fourier transform. We can therefore define fields with continuous Fourier transforms

$$
\begin{aligned}
& \Psi_{1}(\mathbf{r})=\sum_{q} e^{i(Q+q) y} \eta_{1, q} f_{q}(x) \\
& \Psi_{2}(\mathbf{r})=\sum_{q} e^{i(-Q+q) y} \eta_{2, q} f_{-q}(x) .
\end{aligned}
$$

Eq. (33) becomes

$$
\begin{aligned}
& \Psi_{\uparrow}(\mathbf{r})=\Psi_{1}(\mathbf{r})+\Psi_{2}(\mathbf{r}) \\
& \Psi_{\downarrow}^{\dagger}(\mathbf{r})=-\Psi_{1}(\mathbf{r})+\Psi_{2}(\mathbf{r})
\end{aligned}
$$

or in terms of spinor

$$
\tilde{\Psi}(\mathbf{r})=\left(\begin{array}{c}
\Psi_{1}(\mathbf{r}) \\
\Psi_{2}(\mathbf{r})
\end{array}\right)
$$

we have for the spinor Eq. (11)

$$
\Psi(\mathbf{r})=\left(\begin{array}{c}
\Psi_{\uparrow}(\mathbf{r}) \\
\Psi_{\downarrow}^{\dagger}(\mathbf{r})
\end{array}\right)=\left(\begin{array}{cc}
1 & 1 \\
-1 & 1
\end{array}\right) \tilde{\Psi}(\mathbf{r})
$$


The kinetic energy has the form

$$
\mathcal{H}_{K}=\sum_{q} v q\left[\eta_{1, q}^{\dagger} \eta_{1, q}+\eta_{2, q}^{\dagger} \eta_{2, q}\right]=\int d x d y \tilde{\Psi}^{\dagger}(\mathbf{r})\left[-i v \partial_{y}\right] \tilde{\Psi}(\mathbf{r}) .
$$

A crucial observation for QHE is the role of impurities which in general has the form

$$
\mathcal{H}_{i m p}=\int d x d y \Psi^{\dagger}(\mathbf{r}) \tau_{3} \Psi(\mathbf{r}) V(\mathbf{r})
$$

Within the subspace of surface states this interaction becomes, using Eq. (38)

$$
\mathcal{H}_{i m p}=\int d x d y \tilde{\Psi}^{\dagger}(\mathbf{r}) \tau_{1} \tilde{\Psi}(\mathbf{r}) V(\mathbf{r})
$$

This impurity potential can be "gauged" away $\underline{15}$ by a transformation

$$
\tilde{\Psi}(\mathbf{r}) \rightarrow \exp \left[(i / v) \int^{y} V\left(x, y^{\prime}\right) d y^{\prime} \tau_{1}\right] \tilde{\Psi}(\mathbf{r})
$$

which eliminates the impurity potential. Hence transport of chiral states is equivalent to that of a pure system. Chirality implies no channel for backscattering, hence impurities are indeed expected to be ineffective. As noted in section III, (100) surface states with energy $>\Delta^{\prime}$ may mix with bulk states by impurities. The QHE is then limited to temperatures $T<\Delta^{\prime}$.

To evaluate the spin Hall conductance, we define a spin voltage $V_{s}(x)$ ( $\mathrm{x}$ is a coordinate perpendicular to the edge) such that $(\hbar / 2) \rho_{s}(x) V_{s}(x)$ is the coupling energy density to a density $\rho_{s}(x)$ of $\hbar / 2$ spins. This can be represented by a Zeeman term with $V_{s}(x)=e B_{z}(x) / m c$ where $m$ is the electron mass; the corresponding force in the $x$ direction is $(e / m c) d B_{z}(x) / d x$. The unit of spin conduction, in analogy with $e^{2} / h$ of the charge conduction is $(\hbar / 2)^{2} / h=\hbar / 8 \pi$. Hence the spin Hall conductance $\sigma_{x y}^{\text {spin }}=I_{s} / V_{s}$ is

$$
\sigma_{x y}^{\text {spin }}=2 \frac{\hbar}{8 \pi} \operatorname{sign}\left(\Delta \Delta^{\prime}\right)
$$

The thermal Hall conduction is derived in a similar way from the heat conduction of an ideal gas, yielding

$$
\frac{K_{x y}}{T}=\frac{2 \pi^{2} k_{B}^{2}}{3 h} \operatorname{sign}\left(\Delta \Delta^{\prime}\right)
$$

Hence $K_{x y} / T$ is also quantized in this weakly disordered system.

We reconsider now the effect of disorder on the spin Hall conductance. Imagine many random Hall systems, each with their own localized chiral states which are weakly coupled. If the couplings are too weak we expect no currents between the systems so overall $\sigma_{x y}^{\text {spin }}=0$. As the coupling strength increases we expect a finite current to circulate around the ensemble of grains, leading to Eqs. (43144). The transition is in fact induced by disorder: For weak disorder the argument of Eq. (42) holds and the Hall coefficients have their quantized values, Eqs. (43/44). As disorder increases, opposite chiral channels get coupled leading to formation of localized chiral loops which eventually have an insulating behavior, i.e. the Hall conduction vanishes. This quantum Hall plateau transition ${ }^{14}$ has been simulated by a network model, showing a novel type of QHE criticality.

\section{QUANTUM HALL EFFECTS - A BULK APPROACH}

We consider next the effective action of a bulk $d+i d^{\prime}$ superconductor and derive its (charge) Hall conductance $\sigma_{x y}(\mathbf{q}, \omega)$. We assume a thin film situation with the scalar and vector potentials $\phi, \mathbf{A}$ being $z$ independent, as well as $A_{z}=0$. In terms of the Nambu spinors Eq. (2) the off-diagonal Hamiltonian $\int d^{2} r \psi^{\dagger}(\mathbf{r}) h_{\Delta} \psi(\mathbf{r})$ (Eq. A6) is

$$
h_{\Delta}=-\left[\Delta\left(-\partial_{x}^{2}+\partial_{y}^{2}\right) \tau_{1}+\Delta^{\prime} \partial_{x} \partial_{y} \tau_{2}\right] / k_{F}^{2}
$$

and we neglect terms with $\nabla \theta<<k_{F}$. The action in presence of the electromagnetic potentials $\mathbf{A}, \varphi$ is then

$$
\begin{aligned}
S & =\int d^{2} r d t \psi^{\dagger}\left(i \partial_{t}-\tau_{3} \epsilon(\hat{p})-h_{\Delta}-\Sigma\right) \psi \\
\Sigma & =\tau_{3}\left(a_{0}+\mathbf{a}^{2} / 2 m\right)+\mathbf{a} \cdot \mathbf{p} / m-i \boldsymbol{\nabla} \cdot \mathbf{a} / 2 m
\end{aligned}
$$


where $\epsilon(\hat{p})=\left(\hat{p}^{2}-k_{F}^{2}\right) / 2 m$ and we introduce the gauge invariant potentials $\mathbf{a}=\frac{1}{2} \boldsymbol{\nabla} \theta-e \mathbf{A}$ and $a_{0}=\frac{1}{2} \frac{\partial}{\partial t} \theta-e \varphi$. The $\theta$ derivatives arise from the spinor transformation $\Psi(\mathbf{r}) \rightarrow \exp \left[i \tau_{3} \theta(\mathbf{r}) / 2\right] \Psi(\mathbf{r})$. Integrating out the fermions (Appendix B) and expansion to 2 nd order in $\mathbf{a}, a_{0}$ leads to the effective action

$$
\left.\left.S_{e f f}=\int \frac{d^{2} q d \omega}{(2 \pi)^{3}} P_{\mu \nu}(\mathbf{q}, \omega) a_{\mu}(\mathbf{q}, \omega)\right) a_{\nu}(-\mathbf{q}, \omega)\right)
$$

At $T=0$ and $\mathbf{q}, \omega \rightarrow 0$ we obtain $P_{00}=N_{0}$ (density of states which is $N_{0}=m / 2 \pi$ in two dimensions), $P_{i j}=-N_{0} c_{s}^{2}$ where $c_{s}=v_{F} / \sqrt{2}$, while $P_{0 j}(q)=i \operatorname{sign}\left(\Delta \Delta^{\prime}\right) \epsilon_{0 i j} q_{i} /(4 \pi)$ and $\epsilon_{0 i j}$ is the antisymmetric unit tensor. The latter term reflects BTRS and is derived for $\Delta^{\prime} \ll \Delta$.

Integrating out the phase $\theta$ we obtain the effective action in terms of the electromagnetic potentials $\mathbf{A}, \varphi$

$$
\begin{aligned}
& S_{e f f}\{\mathbf{A}, \varphi\}=e^{2} \int \frac{d^{2} q d \omega}{(2 \pi)^{3}}\left\{\quad \frac { c _ { s } ^ { 2 } \mathbf { q } ^ { 2 } } { c _ { s } ^ { 2 } \mathbf { q } ^ { 2 } - \omega ^ { 2 } } \left[P_{00}|\varphi(\mathbf{q}, \omega)|^{2}-\frac{i}{4 \pi} \epsilon_{0 i j} q_{i} \varphi(\mathbf{q}, \omega) A_{j}(-\mathbf{q},-\omega)\right.\right. \\
& \left.\left.+O\left(\omega^{2}|\mathbf{A}|^{2}\right)\right]-P_{00}\left(\frac{c_{s}}{c}\right)^{2}|\mathbf{A}(\mathbf{q}, \omega)|^{2}\right\}
\end{aligned}
$$

The total electromagnetic action includes also the Maxwell terms $S_{M}=\int d^{2} r d t\left(\vec{E}^{2}-\vec{H}^{2}\right) / 8 \pi$. A may also be integrated out, using $\boldsymbol{\nabla} \cdot \mathbf{A}=0$ and $A_{z}=0$ leading to the effective action

$$
\begin{aligned}
\left.S_{e f f}\{\phi)\right\} & =\frac{e^{2}}{\chi(q, \omega)}|\phi|^{2}\left\{\left[\frac{c_{s}^{2} q^{2} P_{00}}{c_{s}^{2} q^{2}-\omega^{2}}+\frac{q^{2} d}{8 \pi e^{2}}\right] \chi(q, \omega)-\frac{1}{(8 \pi c)^{2}} \frac{c_{s}^{4} q^{6}}{\left(c_{s}^{2} q^{2}-\omega^{2}\right)^{2}}\right\} \\
\chi(q, \omega) & =P_{00} \frac{c_{s}^{2}}{c^{2}}+\frac{\omega^{2} q^{2}}{(8 \pi)^{2} c^{2} P_{00}\left(c_{s}^{2} q^{2}-\omega^{2}\right)}+\frac{d \omega^{2}}{8 \pi c^{2} e^{2}}-\frac{d q^{2}}{8 \pi e^{2}}
\end{aligned}
$$

The coefficient of $|\phi|^{2}$ vanishes when $q \rightarrow 0$ at the plasma frequency $\omega_{p}=\left(4 \pi n e^{2} / m\right)^{1 / 2}=c / \lambda_{0}$; there are no acoustic plasmons.

The Hall current $J_{y}$ is identified by a functional derivative with respect to $A_{y}$ leading to the Hall coefficient

$$
\sigma_{x y}(\mathbf{q}, \omega)=\operatorname{sign}\left(\Delta \Delta^{\prime}\right) \frac{e^{2}}{4 \pi \hbar} \frac{c_{s}^{2} q^{2}}{c_{s}^{2} q^{2}-\omega^{2}}
$$

Transport is defined by taking the $q \rightarrow 0$ limit first, i.e. $\sigma_{x y}=0$. Hence the conventional Hall coefficient vanishes, as expected from Galilean invariance ${ }^{16}$. A limit in which $\omega \rightarrow 0$ is taken first yields a quantized "static" conductance $e^{2} / 2 h$ which was argued to correspond to $\sigma_{x y} \neq 0$ in presence of a boundary $\underline{13}$. In absence of an external magnetic field, and given a spontaneous magnetization decaying in the bulk (as shown in section III), Ampére's law yields zero total current, hence $\sigma_{x y}=0$; this is valid also with a boundary and an external electric field. It is intriguing, however, that $\sigma_{x y}(\mathbf{q}, \omega)$ has a nontrivial structure and space resolved measurement of a Hall current could then probe the full Eq. (50). We note that a result similar to Eq. (50) was obtained for superfluid ${ }^{3} \mathrm{He} \underline{17}$.

\section{CONCLUSIONS}

We consider now in more detail the experimental data on the spontaneous magnetization ${ }^{3}$. The data shows that for a YBCO disc with a perimeter of $L_{y} \approx 2 \mathrm{~cm}$ the spontaneous magnetization is temperature independent in the range $80-89 \mathrm{~K}$ and is also thickness independent in the range $30-300 \mathrm{~nm}$ with a value of $\approx 37 \phi_{0}$. Taking $\lambda \Delta \approx \lambda_{0} \Delta_{0}$, their $T=0$ value, and typical YBCO parameters we find $\tilde{\Phi} \approx 10^{-3}$. The temperature and thickness independence indicate weak BTRS with $\xi^{\prime}>\lambda$. For either thick or thin films we estimate $\lambda / \xi^{\prime} \approx 10^{-2}$ or $\Delta^{\prime} / \Delta \approx 10^{-4}$. We propose therefore that increasing the ratio $\Delta^{\prime} / \Delta$, e.g. by using overdoped $\mathrm{YBCO}^{2}$, one can enhance the spontaneous magnetization up to a maximum of $\approx 10^{3} \phi_{0}$ when $\Delta^{\prime} / \Delta \approx 0.01$ within the thick film regime.

For strong BTRS, $\lambda / \xi^{\prime}>1$, the film thickness matters, i.e. we expect a temperature dependence due to the crossover from thick to thin film regimes at $\bar{d} \approx \xi^{\prime}$ as $T \rightarrow T_{c}$. For thin films $\left(\bar{d}<\xi^{\prime}<\lambda\right)$ we obtain $\tilde{\Phi}=\lambda / 12 \xi^{\prime}$, i.e. for YBCO the total flux can reach $10^{5} \Delta^{\prime} / \Delta \phi_{0}$ per cm of boundary, much higher than thick film values. The situation of a strong BTRS with thin films is interesting also as being the most likely one to show the paramagnetic anomaly (Fig. 4) at a temperature $\approx T_{c}\left[1-\left(\xi / \xi^{\prime}\right)^{2}\right]$.

In conclusion, we have shown that surface states of a $d+i d^{\prime}$ superconductor lead to spontaneous magnetization which is $T$ independent and thickness independent for weak BTRS, $\lambda / \xi^{\prime}<1$, in accord with the data ${ }^{3}$. For strong 
BTRS with $\lambda / \xi^{\prime}>1$, as expected in overdoped $\mathrm{YBCO}^{2}$, a crossover from thick to thin film behavior can lead to $T$ and thickness dependence, as well as to an observable paramagnetic anomaly near $T_{c}$. We have shown gapless chiral surface states for the $d+i d^{\prime}$ state which lead to quantization of the spin and thermal Hall conductances. The $d+i s$ state has surface currents only at the (110) surface; its surface excitations have a gap and therefore insulating; i.e. no nontrivial quantization of Hall conductances. For the charge Hall conductance we find a vanishing transport value, however the structure of $\sigma_{x y}(\mathbf{q}, \omega)$ has an unusual form which exhibits the Goldstone mode of the superconductor.

\section{Acknowledgments}

We thank Y. Dagan, G. Deutscher, A. J. Legget, O. Milo, and E. Polturak for valuable discussions. This research was supported by THE ISRAEL SCIENCE FOUNDATION founded by the Israel Academy of Sciences and Humanities.

\section{APPENDIX A: HAMILTONIAN FOR D WAVE SUPERCONDUCTOR}

We derive here the interaction term for a d+id' superconductor. A general interaction Hamiltonian in terms of a Nambu spinor Eq. (2) is

$$
\mathcal{H}_{\text {int }}=-\int \Psi^{\dagger}\left(\mathbf{r}_{1}\right) \tau_{3} \Psi\left(\mathbf{r}_{1}\right) \Psi^{\dagger}\left(\mathbf{r}_{2}\right) \tau_{3} \Psi\left(\mathbf{r}_{2}\right) V\left(\mathbf{r}_{1}-\mathbf{r}_{2}\right)
$$

The order parameter has the form

$$
e^{i \theta(\mathbf{r}) \tau_{3}} \tau_{1} \Delta\left(\mathbf{r}_{1}, \mathbf{r}_{2}\right)=\left\langle\Psi\left(\mathbf{r}_{1}\right) \Psi^{\dagger}\left(\mathbf{r}_{2}\right)\right\rangle V\left(\mathbf{r}_{1}-\mathbf{r}_{2}\right)
$$

where the phase $\theta(\mathbf{r})$ depends only on the center of mass coordinate $\mathbf{r}=\left(\mathbf{r}_{1}+\mathbf{r}_{2}\right) / 2$. The factor $\Delta\left(\mathbf{r}_{1}, \mathbf{r}_{2}\right)$ may be complex, however, its real and imaginary components are determined by the interactions and their ratio is not allowed to vary in space. A d wave superconductor is defined by a momentum dependence $k_{x}^{2}-k_{y}^{2}$ for the relative coordinate, i.e.

$$
\Delta\left(\mathbf{r}_{1}, \mathbf{r}_{2}\right)=\Delta(\mathbf{r}) \int e^{i \mathbf{k} \cdot\left(\mathbf{r}_{1}-\mathbf{r}_{2}\right)}\left(k_{x}^{2}-k_{y}^{2}\right) \frac{d^{2} k}{\left(2 \pi k_{F}\right)^{2}}=\Delta(\mathbf{r})\left(-\partial_{\xi}^{2}+\partial_{\eta}^{2}\right) \delta^{2}(\boldsymbol{\rho}) / k_{F}^{2}
$$

where the relative coordinate is $\boldsymbol{\rho}=\mathbf{r}_{1}-\mathbf{r}_{2}=(\xi, \eta)$. The mean field Hamiltonian is then

$$
\mathcal{H}_{i n t}^{M F}=\int d^{2} r d^{2} \rho \Psi^{\dagger}\left(\mathbf{r}+\frac{1}{2} \boldsymbol{\rho}\right) e^{i \theta(\mathbf{r}) \tau_{3}} \tau_{1} \Psi\left(\mathbf{r}-\frac{1}{2} \boldsymbol{\rho}\right) \Delta(\mathbf{r})\left[\partial_{\xi}^{2}-\partial_{\eta}^{2}\right] \delta^{2}(\mathbf{r}) / k_{F}^{2}
$$

After partial integrations,

$$
\begin{aligned}
\mathcal{H}_{i n t}^{M F}= & \frac{1}{4} \int d^{2} r \Psi^{\dagger}(\mathbf{r})\left[\left(\partial_{x}^{2}-\partial_{y}^{2}\right) \Delta(\mathbf{r}) e^{i \theta(\mathbf{r}) \tau_{3}}\right] \tau_{1} \Psi(\mathbf{r}) / k_{F}^{2} \\
& -\int d^{2} r \Psi^{\dagger}(\mathbf{r})\left[\partial_{x} \Delta(\mathbf{r}) e^{i \theta(\mathbf{r}) \tau_{3}} \partial_{x}-\partial_{y} \Delta(\mathbf{r}) e^{i \theta(\mathbf{r}) \tau_{3}} \partial_{y}\right] \tau_{1} \Psi(\mathbf{r}) / k_{F}^{2}
\end{aligned}
$$

where in the first term $\left(\partial_{x}^{2}-\partial_{y}^{2}\right)$ operates only within the [] brackets. A d' component corresponds to $\Delta^{\prime}(\mathbf{r}) \partial_{\xi} \partial_{\eta} \delta^{2}(\boldsymbol{\rho})$ and similar analysis can be followed. We assume here that all gradients are small, i.e. $|\nabla \theta|,|\nabla \Delta| / \Delta \ll k_{F}$, hence with the transformation $\Psi(\mathbf{r}) \rightarrow \exp \left[i \tau_{3} \theta(\mathbf{r}) / 2\right] \Psi(\mathbf{r})$ yields the off-diagonal Hamiltonian $\int d^{2} r \Psi^{\dagger}(\mathbf{r}) h_{\Delta} \Psi(\mathbf{r})$ where

$$
h_{\Delta}=-\left[\Delta\left(-\partial_{x}^{2}+\partial_{y}^{2}\right) \tau_{1}+\Delta^{\prime} \partial_{x} \partial_{y} \tau_{2}\right] / k_{F}^{2}
$$


The issue of gauge invariance is of some interest. The full interaction form Eq. (A1) is manifestly invariant under $\Psi(\mathbf{r}) \rightarrow \exp \left[i \tau_{3} \int^{\mathbf{r}} A\left(\mathbf{r}^{\prime}\right) \cdot d \mathbf{r}^{\prime}\right] \Psi(\mathbf{r})$. Wether the mean field form is also gauge invariant is a matter of some debate ${ }^{21.22}$. From the definition Eq. A3 it seems that

$$
\Delta\left(\mathbf{r}_{1}, \mathbf{r}_{2}\right) \rightarrow \exp \left[i \tau_{3} \int^{\mathbf{r}_{1}} A\left(\mathbf{r}^{\prime}\right) \cdot d \mathbf{r}^{\prime}\right] \exp \left[i \tau_{3} \int^{\mathbf{r}_{2}} A\left(\mathbf{r}^{\prime}\right) \cdot d \mathbf{r}^{\prime}\right] \Delta\left(\mathbf{r}_{1}, \mathbf{r}_{2}\right)
$$

and then $\mathcal{H}_{i n t}^{M F}$ is gauge invariant without having explicit $A(\mathbf{r})$ dependent terms. This, however, implies that the $\partial_{x}, \partial_{y}$ terms do not follow the usual substitution law as in the kinetic term. For the present work this issue is irrelevant since we neglect these terms altogether, i.e. we assume $|\nabla \theta|,|\nabla \Delta| \ll k_{F}$.

\section{APPENDIX B: DERIVATION OF $P_{i j}$}

We derive here an effective action for a $d+i d^{\prime}$ superconductor in terms of the gauge invariant potentials $a_{\mu}(q, \omega)$, Eq. (47). Integrating out the fermionic variables in the partition function we arrive to the following action:

$$
\begin{aligned}
Z & =\int D \Phi e^{i S} \\
S(\Phi) & =-i \operatorname{Tr} \ln \hat{G}^{-1}, G^{-1}=G_{0}^{-1}-\Sigma \\
G_{0}^{-1} & =i \partial_{t}-\tau_{3} \epsilon(p)-h_{\Delta}
\end{aligned}
$$

We are interested in the long wavelength limit; also the order parameter is taken at the extremum of the effective action with only phase fluctuations. We retain the first and the second order in $\Sigma$ to derive an expansion of the effective action in the fluctuating fields $a_{\mu}(q, \omega)$. The expansion corresponds to a one loop calculation with the coefficients $P_{\mu, \nu}$ in Eq. (47) given by (latin indices stand for space coordinates)

$$
\begin{aligned}
P_{00}(\mathbf{q} \omega) & =\frac{i}{2} T \sum_{\mathbf{p}, \omega^{\prime}} \operatorname{Tr}\left[G\left(p, \omega^{\prime}\right) \tau_{3} G\left(p+q, \omega^{\prime}+\omega\right) \tau_{3}\right] \\
\left.P_{i j} \mathbf{q} \omega\right) & =\frac{i}{2 m^{2}} T \sum_{\mathbf{p}, \omega^{\prime}} \operatorname{Tr}\left[G\left(p, \omega^{\prime}\right) G\left(p+q, \omega^{\prime}+\omega\right) p_{i}(p+q / 2)_{j}\right]-\frac{n}{2 m} \delta_{i, j} \\
P_{0 j}(\mathbf{q} \omega) & =\frac{i}{2 m} T \sum_{\mathbf{p}, \omega^{\prime}} \operatorname{Tr}\left[G\left(p, \omega^{\prime}\right) \tau_{3} G\left(p+q, \omega^{\prime}+\omega\right) p_{j}\right]
\end{aligned}
$$

The diagonal time polarization operator $P_{00}$ depends weakly on temperature and therefore in the limit of small momentum and frequency $q \rightarrow 0, \omega \rightarrow 0$ is given by its $T=0$ value, i.e. the mean-field compressibility, $P_{00}(q)=$ $N_{0}$. The space components $P_{i, j}$ include the diamagnetic term and paramagnetic current correlator. In the limit $q \rightarrow 0, \omega \rightarrow 0$ they give the mean-field superfluid stiffness; at $T \rightarrow 0 P_{i j}(q)=-N_{0} c_{s}^{2}$ where $c_{s}=v_{F} / \sqrt{2}$. Of special significance is the off-diagonal polarization bubble $P_{0, j}$ which is responsible for the Hall effect. It is a topological effect depending (at least at small values of the $d_{x, y}$ order parameter) only on the sign of $\Delta^{\prime}$. In the same long wave-length limit we have $P_{0 j}(q)=i \operatorname{sign}\left(\Delta^{\prime}\right) \epsilon_{0 i j} q_{i} /(4 \pi)$ where $\epsilon_{0 i j}$ is the antisymmetric unit tensor.

\section{APPENDIX C: EFFECTIVE ACTION WITH BOUNDARY}

We study here the Hall term with boundary and show that its effect on London's equation is small at either $T=0$ or $T \rightarrow T_{c}$. The electromagnetic response to the surface charge and currents couples in general the vector and scalar potentials A, $\varphi$ with the Hall coefficient. We estimate this effect first at $T=0$. The Hall term relates the current along the surface ( $y$ direction) and the electric field $\partial \varphi / \partial x$ in the $x$ direction, i.e.

$$
\left(\frac{1}{\lambda_{L}^{2}}-\frac{\partial^{2}}{\partial x^{2}}\right) A_{y}(x)-\frac{4 \pi}{d c} \sigma_{x y} \frac{\partial \varphi}{\partial x}=-\frac{4 \pi}{c} j_{e d g e}(x)
$$

The equation for $\varphi(x)$ involves the Debye screening length $\lambda_{d}=1 / \sqrt{4 m e^{2}} \ll \xi^{\prime}, \xi$,

$$
\left(\frac{1}{\lambda_{d}^{2}}-\frac{\partial^{2}}{\partial x^{2}}\right) \varphi(x)=4 \pi n_{e d g e}(x)
$$


The Hall term is neglected here as we wish to estimate the lowest order effect. The solution with $\partial \varphi / \partial x=0$ involves the Greens' function Eq. [18]; at $x \ll \xi^{\prime}$ it has $\partial \varphi / \partial x \sim \lambda_{d} / \xi^{\prime}$ while at $x \gg \lambda_{d}$

$$
\varphi(x)=-\frac{\lambda_{d}^{2} e k_{F}}{2 \pi d \xi^{\prime}} \int_{0}^{\pi / 2} d \zeta \cos \zeta \sin ^{2} \zeta e^{-2 x \sin \zeta / \xi^{\prime}}
$$

The ratio of $\partial \varphi / \partial x$ and $j_{\text {edge }}$ terms in Eq. (C1) is then $1 /\left(32 \pi k_{F} \xi^{\prime}\right) \ll 1$; for a (110) surface replace $\xi^{\prime} \rightarrow \xi$. Since $k_{F} \xi^{\prime}>k_{F} \xi \gg 1$ is the criterion for excluding the order parameter fluctuations very near $T_{c}$, we can neglect the Hall term in London's equation (C1).

We consider next $T \rightarrow T_{c}$. The polarization function $P_{00}$ (B2) is obtained by replacing $N_{0} \rightarrow N(T)$ where

$$
N(T)=\sum_{p} \frac{|\Delta|^{2}}{2 E^{3}} \tanh \left(\frac{E}{2 T}\right)
$$

with $E=\sqrt{\epsilon^{2}(p)+g_{1}^{2}+g_{2}^{2}}$ where

$$
\begin{aligned}
& g_{1}=\Delta(\mathbf{r}) \cos 2 \zeta \\
& g_{1}=\Delta^{\prime}(\mathbf{r}) \sin 2 \zeta .
\end{aligned}
$$

The polarization function (B3) defines the temperature dependent London penetration depth $1 / \lambda_{L}^{2}(T)$.

We consider in more detail the Chern Simon (or Hall) part of the action which is the product of scalar and vector potentials (Eq. B4). We consider a superconductor that occupies the half space $(x>0)$ where the order parameters $\Delta, \Delta^{\prime}$ may become functions of $x$. The Chern Simon part of the action can be written in configuration space in the form

$$
\begin{aligned}
S_{c-s} & =e^{2} T \sum_{\omega} \int d r\left[b_{1}\left(r r^{\prime}\right) a_{0}(r \omega) a_{y}(r \omega)+b_{2}\left(r r^{\prime}\right) a_{0}(r \omega) \frac{\partial a_{y}(r \omega)}{\partial x}\right]_{r \rightarrow r^{\prime}} \\
b_{1}\left(r r^{\prime}\right) & =\frac{\epsilon_{i j}}{2 m} \sum_{\mathbf{p}} p_{y} \frac{\partial}{\partial x}\left(F_{p}\left(r, r^{\prime}\right) g_{i}(r)\right) \frac{\partial g_{j}}{\partial p_{x}} \\
b_{2}\left(r r^{\prime}\right) & =\frac{\epsilon_{i j}}{2 m} \sum_{\mathbf{p}} p_{y} F_{p}\left(r^{\prime}, r\right) g_{i}\left(r^{\prime}\right) \frac{\partial g_{j}(r)}{\partial p_{x}} \\
F_{p}\left(r r^{\prime}\right) & =\frac{2\left[E^{\prime} t h\left(\frac{E}{2 T}\right)-E t h\left(\frac{E^{\prime}}{2 T}\right)\right]}{E E^{\prime}\left(E^{\prime 2}-E^{2}\right)}
\end{aligned}
$$

where $E=E(r), E^{\prime}=E\left(r^{\prime}\right)$. Similarly as for the infinite system we can integrate out the Goldstone mode

$$
S_{b}\{\mathbf{A}, \varphi\}=e^{2} \int d r\left[P_{00} \varphi^{2}(\mathbf{r})-\frac{c}{4 \pi}\left(\frac{1}{\lambda_{L}(T)}\right)^{2} \mathbf{A}^{2}(\mathbf{r})+b_{1}(\mathbf{r}) \varphi(\mathbf{r}) A_{y}(\mathbf{r})+b_{2}(\mathbf{r}) \varphi(\mathbf{r}) \frac{\partial A_{y}(\mathbf{r})}{\partial x}\right]
$$

In this equation we took the polarization function at zero frequency which is legitimate for finite system (the effective momentum deviates from zero). The coefficients $b_{1}=\left.b_{1}\left(r r^{\prime}\right)\right|_{r \rightarrow r^{\prime}}, b_{2}=\left.b_{2}\left(r r^{\prime}\right)\right|_{r \rightarrow r^{\prime}}$ are given as

$$
\begin{aligned}
4 \pi e^{2} b_{1} & =\frac{e^{2}}{\hbar c d \xi} f_{1}(x, T) \\
4 \pi e^{2} b_{2} & =\frac{e^{2}}{\hbar c d} f_{2}(x, T) .
\end{aligned}
$$

The function $f_{1}$ appears only for a system with boundary and depends on the space derivative of the order parameters, while $f_{2}$ at $T=0$ is the same as for an infinite superconductor. We calculate these function for $T=0$ and for $T \rightarrow T_{c}$,

$$
\begin{aligned}
& f_{1}(x, T)=\xi \frac{d}{d x} \ln \left[\Delta\left(\Delta^{\prime}\right)^{(1+\delta) / 2}\right] \quad \text { if } T=0 \\
& =0.11 \xi \frac{\Delta \Delta^{\prime}}{T_{c}^{2}} \frac{d}{d x} \ln \left[\Delta \Delta^{\prime}\right] \quad \text { if } T \rightarrow T_{c} \\
& f_{2}(x, T)=\quad 1 \quad \text { if } T=0 \\
& =\quad 0.21 \frac{\Delta \Delta^{\prime}}{T_{c}^{2}} \quad \text { if } T \rightarrow T_{c}
\end{aligned}
$$


with $\delta=2 /\left(1+\Delta^{\prime} / \Delta\right)$ and $\Delta(x), \Delta^{\prime}(x)>0$ is assumed. Thus, in the limit $T \rightarrow T_{c}$ we we can write $\lambda \approx \lambda_{0} / \sqrt{\tau}$, $b_{1}(\mathbf{r})=0.11\left(\Delta \Delta^{\prime} / T_{c}^{2}\right) \frac{d}{d x} \ln \left[\Delta \Delta^{\prime}\right] / 2 h c d$ and $b_{2}(\mathbf{r})=0.21\left(\Delta \Delta^{\prime} / T_{c}^{2}\right) / 2 h c d$.

The next step involved the equations for electromagnetic potentials - generalized London equations by the variations of the total action (including the Maxwell part) over these potentials. We consider here the half-plane geometry, i.e. the superconductor occupies the $x>0$ half-plane. The nonzero electromagnetic potentials $A_{y}, \varphi$ obey the equations

$$
\begin{aligned}
\left(\frac{1}{\lambda_{d}^{2}}-\frac{\partial^{2}}{\partial x^{2}}\right) \varphi-\frac{4 \pi e^{2}}{c \hbar} b_{2} \frac{\partial A_{y}}{\partial x}-\frac{4 \pi e^{2}}{c \hbar} b_{1} A_{y} & =4 \pi e n_{\text {edge }}(x) \\
\left(\frac{1}{\lambda_{L}^{2}}-\frac{\partial^{2}}{\partial x^{2}}\right) A_{y}-\frac{4 \pi e^{2}}{c \hbar} b_{2} \frac{\partial \varphi}{\partial x}+\frac{4 \pi e^{2}}{c \hbar}\left(b_{1}-\partial_{x} b_{2}\right) \varphi & =-\frac{4 \pi}{c} j_{\text {edge }}(x)
\end{aligned}
$$

where $n_{\text {edge }}, j_{\text {edge }}$ are the edge charge and edge current densities (Eq. [16).

Using the expressions above for $b_{1}, b_{2}$ we find that the Chern-Simon term affects the spontaneous magnetization, leading to an additional flux $\sim\left(\Delta^{\prime} / \Delta\right) \tau$ which vanishes at $T \rightarrow T_{c}$, i.e. it is negligible compared with the other terms in Eq. (C17) which lead to constant magnetization as $T \rightarrow T_{c}$, as shown in section III.

1 M. Covington et al., Phys. Rev. Lett. 79, 277 (1997).

2 G. Deutscher, Y. Dagan, A. Kohen and R. Krupke, Physica C 341-348 1629 (2000); Phys. Rev. Lett. 87, 177004 (2001); Phys. Rev. B 64, 092509 (2001).

3 R. Carmi, E. Polturak, G. Koren, and A. Averbach, Nature 404, 853 (2000)

4 Alff L. et al. European Phys. J. B 5, 423 (1998).

5 A. Sharoni, G. Koren and O. Millo, Europhysics Letts. 54, 675 (2001) ; A. Sharoni et al. cond-mat/0111156 (2001)] Phys. Rev. B 65134526 (2002).

6 M. Sigrist, D. B. Bailey and R. B. Laughlin, Phys. Rev. Lett. 74, 3249 (1995).

7 M. Matsumoto and H. Shiba, J. Phys. Soc. Jpn. 643384 (1995); 644867 (1995).

8 M. Fogelstrom, D. Rainer, and J. A. Sauls, Phys. Rev. Lett. 79, 281 (1997)

9 Š. Kos, Phys. Rev. B 63214506 (2001).

10 Walter H. et al., Phys. Rev. Lett. 80, 3598 (1998).

11 S. Higashitani, J. Phys. Soc. Japan 66, 2556 (1997).

12 Yu S. Barash, M. S. Kalenkov and J. Kurkijärvi, Phys. Rev. B 62, 6665 (2000).

13 J. Goryo and K. Ishikawa, Phys. Letts. A260, 294 (1999).

14 V. Kagalovsky, B. Horovitz, Y. Avishay and J. T. Chalker, Phys. Rev. Lett 82, 3516 (1999).

15 T. Senthil, J. B. Marston and M. P. A. Fisher, Phys. Rev. B Phys. Rev. B60, 4245 (1999).

16 N. Read and D. Green, cond-mat/9906453 Phys. Rev. B 6110267 (2000).

17 J. Goryo and K. Ishikawa, Phys.Lett. A 246, 549 (1998).

18 B. Horovitz and A. Golub, Europhys. Lett. 57, 892 (2002).

19 A. Furusaki, M. Matsumoto and M. Sigrist cond-mat/0102143 Phys. Rev. B 64, 054514 (2001)

20 G. E. Volovik, JETP Lett. 66, 522 (1997).

21 S. H. Simon and P. A. Lee, Phys. Rev. Lett. 781548 (1997).

22 O. Vafek, A. Melikyan, M. Franz and Z. Tešanović, Phys. Rev. B 63134509 (2001). 